\title{
The Effect of Free Radical Enhancers and Scavengers on Accumulation of Early Light-Inducible Protein during Light Stress
}

\author{
Iwona Adamska, Klaus Kloppstech \\ Institut für Botanik, Universität Hannover, Herrenhäuser Straße 2, \\ D-W-3000 Hannover 21, Bundesrepublik Deutschland \\ and \\ Itzhak Ohad \\ Department of Biological Chemistry, The Hebrew University of Jerusalem, \\ Jerusalem 91904, Israel \\ Z. Naturforsch. 48c, 391-396 (1993); received November 3, 1992 \\ Chlorophyll Triplet, D 1 Turnover, Hydroxyl Radicals, Light Stress, Pea Plants \\ Involvement of active oxygen species in the accumulation of early light-inducible protein \\ (ELIP) in mature pea plants was investigated under light stress conditions. Here we provide \\ evidence that hydroxyl radicals and singlet oxygen interfere with ELIP expression at both, \\ transcriptional and posttranslational levels. Addition of free radical scavengers can alleviate \\ the detrimental action of the radicals almost completely at the level of transcription but only a \\ partial protection was observed at the level of proteins. The data suggest that the damage of \\ ELIP occurs during its insertion into thylakoid membranes close to the free radical sources.
}

\section{Introduction}

The nuclear-encoded, thylakoid bound early light-inducible proteins (ELIPs) reported to be expressed during the first hours of the greening process $[1,2]$ are synthesized in substantial amounts in leaves of mature plants exposed to light stress $[3,4]$.

Sequencing of ELIP cDNA clones of pea and barley $[5,6]$ has demonstrated that ELIPs are related to the light-harvesting chlorophyll $a / b(c a b)$ gene family. Based on hydropathy plots of the predicted amino acid sequences of ELIP clones it appears that ELIP exhibits three transmembrane loops out of which loop I and III show a high homology with those of all known $c a b$ gene products [6]. So far no definite function was described for ELIP.

Induction of a protein apparently related to ELIP was reported for the green unicellular alga Dunaliella bardawil $\lceil 7\rceil$ in connexion with $\beta$-caro-

\footnotetext{
Abbreviations: ELIP, early light-inducible protein; LHCP, light-harvesting chlorophyll $a / b$ protein; PAGE, polyacrylamide gel electrophoresis; Phe, pheophytin; PS II, photosystem II; $Q_{A}$, first quinone acceptor; $Q_{B}$, second quinone acceptor.

Reprint requests to I. Adamska.

Verlag der Zeitschrift für Naturforschung,

D-W-7400 Tübingen

0939-5075/93/0300-0391 \$01.30/0
}

tene accumulation under light stress conditions. Inhibition of the carotenoid biosynthesis by the bleaching herbicide fluridone greatly enhances ELIP accumulation in thylakoids of mature pea plants during light stress [8]. A desiccation-related ELIP-like protein has been described for Craterostigma plantagineum [9] which might indicate occurrence of light stress under conditions of desiccation.

When oxygen-evolving photosynthetic organisms utilize water as an electron source and reduce oxygen by the Mehler reaction, they must suppress the release of reactive intermediates of reduced dioxygen such as the superoxide radical $\left(\mathrm{O}_{2}{ }^{-}\right)$, hydroxyl radical $(\cdot \mathrm{OH})$, hydrogen peroxide $\left(\mathrm{H}_{2} \mathrm{O}_{2}\right)$ and excited singlet oxygen $\left({ }^{1} \mathrm{O}_{2}\right)$. Organic derivatives corresponding to reduced species of oxygen are produced in cells, mainly in the form of oxidized unsaturated fatty acids. These include lipid peroxy radical (LOO'), hydroperoxide $(\mathrm{LOOH})$ and alkoxyl radical ( $\left.\mathrm{LO}^{*}\right)$. The lifetimes of these lipid derivatives are generally longer then that of "free" active oxygen.

In the chloroplasts the major sources of the hydroxyl radical and singlet oxygen are their production from superoxide or hydrogen peroxide. In vivo, $\mathrm{Fe}$ (III) is the main participant in the metalcatalyzed formation of hydroxyl radicals but $\mathrm{Cu}(\mathrm{II})$ might also play a minor but definite role. In addition to these metals $\mathrm{Ti}$ (III) or Co(II) are able 
to reduce hydrogen peroxide in vitro producing hydroxyl radicals $[10,11]$. The presence of chlorophyll molecules in the vicinity of the water oxidation complex of PS II makes the thylakoid membranes a potential source for generation of singlet oxygen [12]. The release of chlorophyll and its degradation products enhance the production of singlet oxygen in chloroplasts $[12,13]$.

The oxygen-free radicals interact with proteins and other target molecules, including DNA, to induce cellular damage [14]. The primary cellular defense against the generated radicals are the enzyme systems like superoxide dismutase, catalase, ascorbate peroxidase or glutathione peroxidase [15]. Oxygen-free radicals also may be detoxified nonenzymatically, particularly in chloroplasts through the action of ferredoxin [16], metallothionine, and $\alpha$-tocopherol [17]. Most of the triplet chlorophyll and singlet oxygen are scavenged in the thylakoid membranes by carotenoids [18].

Since ELIPs are induced under conditions where the production of active oxygen species is enhanced it was of interest to test whether free radicals influence ELIP induction. In this work we provide indirect evidence for destructive action of hydroxyl radicals and singlet oxygen on ELIP transcription and accumulation of the protein in the thylakoid membranes.

\section{Materials and Methods}

\section{Growth of plants and illumination}

Pea plants (Pisum sativum L., cv. Alaska) were grown for 14 days on vermiculite at $25^{\circ} \mathrm{C}$ and at the light intensity of $40 \mu \mathrm{E} / \mathrm{m}^{2}$ s provided by white fluorescent lamps, under a light regime of $12 \mathrm{~h}$ dark: 12 h light.

The high light treatment was performed on detached, mature leaves floated on water. High intensity white light was provided by tungsten halogen lamps $(2000 \mathrm{~W})$ yielding $3000 \mu \mathrm{E} / \mathrm{m}^{2} \mathrm{~s}$. The plants were protected from excess infra-red radiation by a $3 \mathrm{~cm}$ double-walled glass screen cooled by a water-circulating system.

\section{Isolation of poly $(A+)-R N A$ and dot blot hybridization}

Poly(A+)-RNA was prepared according to Logemann et al. [19] using oligo(dT)-cellulose chromatography [20]. Dot blot hybridization with the ${ }^{32} \mathrm{P}$-labeled insert was performed as described previously [21]. Insert from an ELIP cDNA clone [5] was labeled using random oligonucleotides as primers according to Boehringer Mannheim protocol.

In vivo labeling and isolation of thylakoid membrane proteins, electrophoresis and immunoblotting

Detached leaves were floated on water with addition of $100 \mu \mathrm{Ci} / \mathrm{ml}\left[{ }^{35} \mathrm{~S}\right]$ methionine $(1220 \mathrm{Ci} /$ mmol, Du Pont) during the light treatment.

Thylakoid membranes were isolated as described [22]. SDS-PAGE was performed according to Laemmli [23] using the Hoeffer minigel system. Radioactive gels treated for fluorography [24] were exposed to X-ray film at $-70{ }^{\circ} \mathrm{C}$. Immunoblotting was carried out according to Towbin et al. [25]. Blots were incubated with antibodies and detected by the enhanced chemiluminescence method (Amersham).

\section{Results}

It has been reported recently [3] that light stress induces ELIP transcription and accumulation of the protein in the thylakoid membranes. It is known that during illumination of plants with high intensity light the photoproduction of active oxygen increases in chloroplasts [12]. To assess whether oxygen-free radicals generated under such conditions might influence accumulation of ELIP the following experiment was performed. Detached green pea leaves were exposed to strong light $\left(3000 \mu \mathrm{E} / \mathrm{m}^{2} \mathrm{~s}\right)$ for different times in absence or presence of the hydroxyl radical scavenger propyl gallate. Thylakoid membranes were isolated and ELIP and D 1 protein levels assayed by immunoblotting (Fig. 1). As shown, ELIP is induced during light stress and accumulates in the thylakoid membranes in a time-dependent manner. The increase in the ELIP level proceeds in parallel with the decrease in the amount of D 1 protein as was already reported [3]. Addition of propyl gallate during illumination enhances ELIP accumulation in the thylakoids and slows down the decrease in D 1 protein level. These results indicate that hydroxyl radicals generated during light stress exert a detrimental effect on the accumulation of ELIP in the thylakoids and influence the steady state level of D 1 protein. The partial inhibition of D 1 degra- 


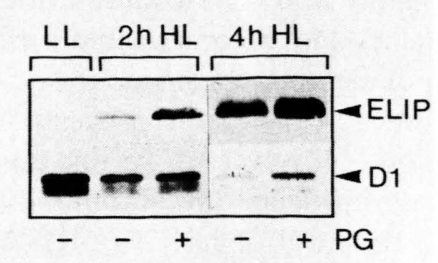

Fig. 1. Free hydroxyl radicals generated during light stress reduce ELIP level in the thylakoid membranes. Detached leaves from plants grown under low light conditions (LL; $40 \mu \mathrm{E} / \mathrm{m}^{2}$ s) were exposed to light stress (HL; $3000 \mu \mathrm{E} / \mathrm{m}^{2} \mathrm{~s}$ ) for different times in presence $(+)$ or absence $(-)$ of $0.5 \mathrm{~mm}$ hydroxyl radical scavenger, propyl gallate $(\mathrm{PG})$. Thylakoid membranes were isolated and level of ELIP and D 1 protein assayed by western blotting.

dation by propyl gallate was reported by Sopory et al. [26].

To investigate at which level the light-generated free radicals affect ELIP expression the action of hydroxyl radicals and singlet oxygen on accumulation of ELIP transcript and protein in the thylakoids were investigated. Since the lifetime of singlet oxygen in illuminated thylakoids is very short $\mathrm{D}_{2} \mathrm{O}$ was used to stabilize the radicals and promote its deleterious effects [27]. To scavenge singlet oxygen selenomethionine was added to the leaves during illumination $[26,28]$. An enhanced level of hy-

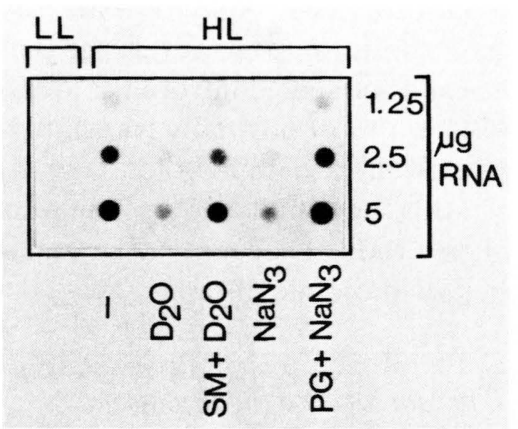

Fig. 2. Effect of oxygen-free radical enhancers and scavengers on accumulation of ELIP transcript. Detached leaves were exposed to light stress (HL; $3000 \mu \mathrm{E} / \mathrm{m}^{2} \mathrm{~s}$ ) for $2 \mathrm{~h}$ in absence $(-)$ or presence of singlet oxygen stabilizer $\mathrm{D}_{2} \mathrm{O}(90 \%)$ alone or in combination with quencher selenomethionine (SM, $2 \mathrm{mM}$ ) or to free hydroxyl radical increaser $\mathrm{NaN}_{3}(10 \mathrm{~mm})$ in combination with scavenger propyl gallate (PG, $0.5 \mathrm{~mm})$. Poly $(\mathrm{A}+)$-RNA was isolated and used for dot blot hybridization with labeled insert of ELIP cDNA clone. Lane LL, poly $(\mathrm{A}+)$-RNA isolated before high light illumination. droxyl radicals was achieved by addition of $\mathrm{NaN}_{3}$. Detached leaves were exposed to light stress in absence or presence of radicals enhancers in combination with radical scavengers and the level of ELIP transcript was tested by dot blotting (Fig. 2). Increased levels of both, hydroxyl radicals and singlet oxygen, repress accumulation of ELIP transcript to $20 \%$ of the untreated control. This effect is alleviated by the use of radical scavengers when added simultaneously with enhancers of radical formation. In such plants the level of ELIP transcript is almost the same as in control plants.

The effects of radical enhancers and scavengers were also tested at the protein level in leaves exposed to low or high light conditions. Addition of radical enhancers to the leaves incubated at low light intensities has no effect on the levels of ELIP and D 1 protein (Fig. 3, left panel); this could be expected since radicals are generated preferentially during exposure of plants to light stress conditions. Under high light conditions (Fig. 3, right panel) the level of ELIP in the thylakoid is lower in presence of hydroxyl radicals and singlet oxygen, however, the action of hydroxyl radicals seems to be more effective. In their presence the level of ELIP in the thylakoids is almost undetectable and this effect can be alleviated only partially by propyl gallate. Also the D 1 protein seems to be more sensitive to the action of hydroxyl radicals than to

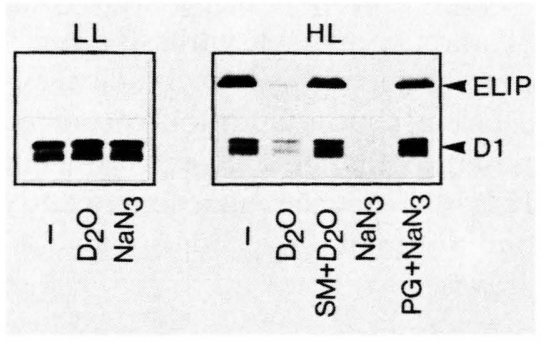

Fig. 3. Effect of oxygen-free radical enhancers and scavengers on accumulation of ELIP in the thylakoid membranes. Detached leaves were exposed to low light (LL; $\left.40 \mu \mathrm{E} / \mathrm{m}^{2} \mathrm{~s}\right)$ or high light $\left(\mathrm{HL} ; 3000 \mu \mathrm{E} / \mathrm{m}^{2} \mathrm{~s}\right)$ for $2 \mathrm{~h}$ in absence $(-)$ or presence of singlet oxygen stabilizer $\mathrm{D}_{2} \mathrm{O}$ $(90 \%)$ alone or in combination with quencher selenomethionine (SM, $2 \mathrm{~mm}$ ) or to free hydroxyl radical enhancer $\mathrm{NaN}_{3}(10 \mathrm{~mm})$ alone or in combination with scavenger propyl gallate (PG, $0.5 \mathrm{~mm})$. Thylakoid membranes were isolated and used for western blotting. 


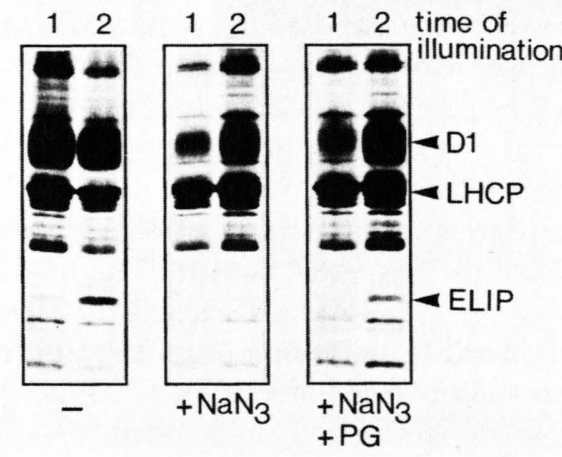

Fig. 4. Effect of free hydroxyl radicals on thylakoid membrane proteins. Detached leaves were exposed to light stress $\left(3000 \mu \mathrm{E} / \mathrm{m}^{2} \mathrm{~s}\right)$ for different times in presence of $100 \mathrm{mCi} / \mathrm{ml}\left[{ }^{35} \mathrm{~S}\right] \mathrm{methionine}$. Free hydroxyl radicals were generated by addition of $10 \mathrm{~mm} \mathrm{NaN}_{3}$ and scavenged by $0.5 \mathrm{~mm}$ propyl gallate. Thylakoid membranes were isolated, separated by SDS-PAGE and the gel used for fluorography. D 1 protein, LHCP and ELIP were identified by western blotting. In left panel, line 2 less proteins were loaded.

singlet oxygen. However, in this case the addition of radical scavengers inhibits completely the deleterious effect.

To proof whether the level of other thylakoid membrane proteins might also be influenced by radicals an in vivo labeling experiment was performed. Detached leaves were exposed to light stress in presence of $\left[{ }^{35} \mathrm{~S}\right]$ methionine without or with addition of sodium azide in combination with propyl gallate. Fluorography (Fig. 4) shows that the rates of ELIP and D 1 protein synthesis are significantly lower in presence of hydroxyl radical enhancer and increase after the addition of propyl gallate. As opposed to that the incorporation of label into LHCP did not change significantly under these conditions and was comparable to that of control leaves.

\section{Discussion}

Free oxygen radicals affect ELIP expression at both, transcriptional and posttranslational levels

In this work we show that accumulation of ELIP transcript is inhibited in presence of free hydroxyl radicals and singlet oxygen as compared with control plants. Radical scavengers applied to such leaves during light stress can reverse this process almost completely and the accumulation of ELIP transcript reaches a level comparable with that of controls. It is not clear at which step the action of free radicals interferes with the process of transcript accumulation. However, it seems unlikely that free radicals influence the stability of ELIP transcript since the level of LHCP mRNA did not change under these conditions (data not shown). It is possible that the damage occurs at the DNA level since it is known that oxygen free radicals cause DNA base pair substitutions and frame shifts as observed in bacterial mutagenesis tests [29] and can cause DNA strand breaks [30].

The detrimental effect of oxygen-free radicals occurs also at the protein level. Under conditions where the level of radicals is enhanced by their stabilizers the amount of ELIP in the thylakoid membranes does not correspond to the amount of its mRNA; while the protein is almost undetectable the mRNA level is only reduced to $20 \%$. Also the protection of the protein by addition of radical scavengers is only a partial one $(50-60 \%)$ while the mRNA level is completely retained. The possibility that under these conditions ELIP mRNA might be damaged was excluded by in vitro translation in the wheat germ system (data not shown). The reduction of ELIP level in the thylakoids could be due to the fact that the translation capacity of the cells is diminished by a high level of free radicals or that transport of the nuclear-encoded proteins into the chloroplasts might be partially arrested under these conditions. However, the fact that another nuclear-encoded, chloroplast localized protein, LHCP, is almost normally translated, transported and inserted into thylakoids (Fig. 4) does not support this possibility. This suggests that the action of free radicals occurs posttranslationally within the thylakoid membranes.

\section{Insertion of ELIP precursor into the thylakoids occurs close to the free radical sources}

Hydroxyl radicals have very high reactivity with almost all cell components at nearly diffusion controlled rates. Thus a hydroxyl radical shows a "site"-specific effect and interacts with cell components only at the site where it is produced [31]. Also singlet oxygen has a very short lifetime and shows high reactivity with surrounding molecules. As shown in this work (Fig. 1) addition of hy- 
droxyl radical scavenger to the leaves during light stress increases the amount of ELIP in the thylakoids. This indicates that under light stress conditions hydroxyl radicals cause damage of the proteins in the thylakoid membranes. It was reported that histidine, cysteine, tyrosine, tryptophan and methionine residues are susceptible to oxidation by both singlet oxygen and hydroxyl radicals. After attack of active oxygen, proteins are more sensitive to proteolytic hydrolysis $[32,33]$. In the previous study [34] it was reported that once induced ELIP is stable and does not turn over significantly under high light conditions. From these facts one can conclude that the detrimental effect of free radicals on ELIP accumulation occurs in the place of its insertion into the membranes, where the damaged protein is immediately degraded. Once inserted ELIP appears to be protected from the action of free radicals, however, the mechanism(s) of this protection remains to be investigated.

Production of hydroxyl radicals in chloroplasts is catalyzed by transition metals [12]. The forms of metal which participate in the production of hydroxyl radicals in chloroplasts are still unknown. However, its production is enhanced by the addition of $\mathrm{Cu}(\mathrm{II})$ as determined by lipid peroxidation [35] or by addition of ferredoxin [36]. One should notice that iron is a central binding ligand in the PS II reaction center [37]. If the site of $Q_{B}$ is modified under light stress conditions such as to prevent electron donation from $Q_{A}$ to $Q_{B}$ the position of $\mathrm{Fe}(\mathrm{II})$ may be affected and $\mathrm{Fe}(\mathrm{III})$ can be formed. $\mathrm{Fe}$ (III) is more harmful and causes the damage of protein and/or pigment components in the immediate vicinity of its formation site. Propyl gallate stabilizes proteins by complexing with metal ions such as iron or copper thereby preventing these metal ions from participation in radical generation [38]. In this context it is of interest to mention that under high light conditions in vitro imported ELIP interacts with components of PS II reaction center, especially with the D 1 protein as shown by crosslinking experiments [22]. Direct production of active oxygen from triplet excited chlorophyll has been shown under aerobic conditions [39]. The reason for D 1 and ELIP degradation may be generation of singlet oxygen, formed by chlorophyll $\mathrm{P}_{680}$ triplet. Under high light conditions double reduced $\mathrm{Q}_{\mathrm{A}}$ is stabilized by protonation and a protein conformational changes followed by release of $\mathrm{Q}_{\mathrm{A}} \mathrm{H}_{2}$ from its binding site [40]. Double reduction of $Q_{A}$ or an empty $Q_{A}$ site is known to facilitate the light-induced formation of a chlorophyll triplet due to recombination of the primary charge pair, $\mathrm{P}_{680+} \mathrm{Phe}^{-}$which formation is normally inhibited by the repulsion between $\mathrm{Q}_{\mathrm{A}}^{-}$and $\mathrm{Phe}^{-}$[41]. The action of propyl gallate which interacts with iron may prevent the damage of proteins by blocking the double reduction of $Q_{A}$. This aspect of interaction is under investigation.

\section{Acknowledgements}

We thank Prof. F. Herzfeld (Institute of Botany, University of Hannover, Germany) for anti-ELIP antibody. This work was supported by a stipendium to I.A. from the MINERVA Foundation (Heidelberg, Germany) and the research grants from Sonderforschungsbereich (Grant SFB-184 awarded to I.O. in cooperation with R. G. Herrmann and W. Rüdiger, Institute of Botany, Munich University, Germany), by the United StatesIsrael Agriculture Binational Foundation (BARD) (to I.O. and W. Vermaas, University of Arizona, U.S.A.) and by the German-Israel Foundation (GIF awarded to I.O. in cooperation with D. Godde, Department of Plant Biochemistry, The Ruhr University, Bochum, Germany). 
[1] G. Meyer and K. Kloppstech, Eur. J. Biochem. 138, 201-207 (1984).

[2] B. Grimm and K. Kloppstech, Eur. J. Biochem. 167, 493-499 (1987).

[3] I. Adamska, I. Ohad, and K. Kloppstech, Proc. Natl. Acad. Sci. U.S.A. 89, 2610-2613 (1992).

[4] E. Pötter and K. Kloppstech, in: Research in Photosynthesis (N. Murata, ed.), Vol. III, pp. 231-238, Kluver Academic Publishers, The Netherlands 1992.

[5] C. Scharnhorst, H. Heinze, G. Meyer, W. Kolanus, K. Bartsch, S. Heinrichs, T. Gutschun, M. Möller, and F. Herzfeld, Plant Mol. Biol. 4, 241-245 (1985).

[6] B. Grimm, E. Kruse, and K. Kloppstech, Plant Mol. Biol. 13, 583-593 (1989).

[7] A. Lers, H. Levy, and A. Zamir, J. Biol. Chem. 266, $13698-13705$ (1991)

[8] I. Adamska, K. Kloppstech, and I. Ohad, J. Biol. Chem. 267, 24732-24737 (1992).

[9] D. Bartels, C. Hanke, K. Schneider, D. Michel, and F. Salamini, EMBO J. 11, 2771-2778 (1992)

[10] J. M. C. Gutteridge and J. V. Bannister, Biochem. J. 234, 225-228 (1986).

[11] B. Halliwell and J. M. C. Gutteridge, Arch. Biochem. Biophys. 246, 501-514 (1986).

[12] K. Asada and M. Takahashi, in: Photoinhibition (D. J. Kyle, C. B. Osmond, and C. J. Arntzen, eds.), pp. 227-287, Elsevier Science Publishers B.V. (Biomedical Division) 1987.

[13] S. Kimura, T. Isobe, H. Sai, and Y. Takahashi, in: Lipid peroxides in Biology and Medicine (K. Yagi, ed.), pp. 243-254, Academic Press, New York N.Y. 1982.

[14] K. Yagi, Lipid Peroxides in Biology and Medicine (K. Yagi, ed.), Academic Press, New York N.Y. 1982.

[15] J. G. Scandalios, Curr. Topics Biolog. and Med. Res. 14, 19-44 (1987).

[16] J. F. Allen, Biochem. Biophys. Res. Commun. 66, 36-43 (1975)

[17] J. Soll, R. Dolce, and G. Schultz, FEBS Lett. 112, 243-248 (1980).

[18] B. Demmig-Adams, Biochim. Biophys. Acta 1020, 1-24 (1990).

[19] J. Logemann, J. Schell, and L. Willmitzer, Anal. Biochem. 163, 16-29 (1987).

[20] R. E. Pemberton, P. A. Liberti, and C. Baglioni, Anal. Biochem. 66, 18-28 (1975).
[21] B. Otto, B. Grimm, P. Ottersbach, and K. Kloppstech, Plant Physiol. 88, 21-25 (1988).

[22] I. Adamska and K. Kloppstech, Plant Mol. Biol. 16, 209-223 (1991)

[23] U. K. Laemmli, Nature 227, 680-685 (1970).

[24] W. M. Bonner and R. A. Laskey, Eur. J. Biochem. 46, 83-88 (1974)

[25] H. Towbin, T. Staehelin, and J. Gordon, Proc. Natl. Acad. Sci. U.S.A. 76, 4350-4354 (1979).

[26] S. K. Sopory, B. M. Greenberg, R. A. Mehta, M. Edelman, and A. K. Mattoo, Z. Naturforsch. 45c, 412-417 (1990).

[27] D. R. Kearns, in: Singlet Oxygen (H. H. Wasserman and R. W. Murray, eds.), pp. 115-137, Academic Press, New York 1979

[28] A. L. Tappel, Fed. Proc. 24, 73-78 (1965).

[29] C. S. Moody and H. M. Hassan, Proc. Natl. Acad. Sci. U.S.A. 79, 2855-2859 (1982)

[30] H. C. Birnboim and M. Kanabus-Kaminska, Proc. Natl. Acad. Sci. U.S.A. 82, 6820-6824 (1985).

[31] R. J. Youngman, Trends Biochem. Sci. 9, 280-283 (1984).

[32] E. R. Stadtman, Trends Biochem. Sci. 11, 11-12 (1986).

[33] S. P. Wolff, A. Gardner, and R. T. Dean, TIBS 11, 27-31 (1986).

[34] J. Adamska, K. Kloppstech, and I. Ohad, J. Biol. Chem. 268, in press (1993)

[35] G. Sandmann and P. Böger, Plant Physiol. 66, 161$165(1980)$

[36] J. R. Bowyer and P. Camilleri, Biochim. Biophys. Acta 808, 235-242 (1985)

[37] O. Prasil, N. Adir, and I. Ohad, Top. Photosynth. 43, 295-348 (1992).

[38] K. J. A. Davies, A. Sevanian, S. F. MuakkassahKelly, and P. Hochstein, Biochem. J. 235, 747-752 (1986).

[39] J. R. Harbour and J. R. Bolton, Photochem. Photobiol. 28, 231-235 (1978).

[40] I. Vass, S. Styring, T. Hundal, A. Koivuniemi, E.-M. Aro, and B. Andersson, Proc. Natl. Acad. Sci. U.S.A. 89, $1408-1412$ (1992).

[41] F. J. E. van Mieghem, W. Nitschke, P. Mathis, and A. W. Rutherford, Biochim. Biophys. Acta 977, 207-214 (1989). 